\title{
Optimization of a sustainable closed loop supply chain network design under uncertainty using multi-objective evolutionary algorithms
}

\author{
Pourjavad, E. , Mayorga, R.V. , $^{\text {a }}$ \\ ${ }^{a}$ Industrial Systems Engineering, University of Regina, Regina, Canada
}

\section{A B S T R A C T}

Environmental, social and economic concerns have highlighted the importance of closed loop supply chain (CLSC) network design problem according to sustainable development. In addition, the uncertainty in decision elements adds to the complexity of this problem. Hence, this paper aims to propose a fuzzy multi-objective mixed integer linear programming (FMOMILP) model for a multi-echelon and multi-period CLSC network that minimize cost and environmental effects and maximize social impacts, simultaneously. At first, the model is converted into a multi-objective mixed-integer linear programming (MOMILP) model by the weighted average method. Due to NPhardness of the problem, a non-dominated sorting genetic algorithm-II (NSGA-II) is developed to solve this multi-objective mathematical model. The obtained results are validated with the non-dominated ranking genetic algorithm (NRGA), due to there is no benchmark for this problem. In addition, different numerical instances are presented and analyzed with different measures in order to indicate the efficiency of proposed algorithms. The provided results demonstrate that the proposed NSGA-II algorithm is an adequate tool to solve the multi-objective problem of CLSC network design.
\end{abstract}

\section{ARTICLE INFO}

\section{Keywords:}

Closed loop supply chain;

Evolutionary algorithms;

Network design;

Sustainability;

Multi-objective optimization

*Corresponding author:

rene.mayorga@uregina.ca

(Mayorga, R.V.)

Article history:

Received 20 December 2017

Revised 1 June 2018

Accepted 5 June 2018

\section{References}

[1] Srivastava, S.K. (2007). Green supply-chain management: A state-of-the-art literature review, International Journal of Management Reviews, Vol. 9, No. 1, 53-80, doi: 10.1111/i.1468-2370.2007.00202.x.

[2] Chen, C.-L., Lee, W.-C. (2004). Multi-objective optimization of multi-echelon supply chain networks with uncertain product demands and prices, Computers \& Chemical Engineering, Vol. 28, No. 6-7, 1131-1144, doi: 10.1016/j. compchemeng.2003.09.014.

[3] Pishvaee, M.S., Rabbani, M., Torabi, S.A. (2011). A robust optimization approach to closed-loop supply chain network design under uncertainty, Applied Mathematical Modelling, Vol. 35, No. 2, 637-649, doi: 10.1016/j.apm. 2010.07.013.

[4] Zhang, Y.M., Huang, G.H., He, L. (2011). An inexact reverse logistics model for municipal solid waste management systems, Journal of Environmental Management, Vol. 92, No. 3, 522-530, doi: 10.1016/i.jenvman.2010.09.011.

[5] Kerachian, R., Karamouz, M. (2007). A stochastic conflict resolution model for water quality management in reservoir-river systems, Advances in Water Resources, Vol. 30, No. 4, 866-882, doi: 10.1016/j.advwatres.2006. $\underline{07.005}$.

[6] Vahdani, B., Jolai, F., Tavakkoli-Moghaddam, R., Mousavi, S.M. (2012). Two fuzzy possibilistic bi-objective zeroone programming models for outsourcing the equipment maintenance problem, Engineering Optimization, Vol. 44, No. 7, 801-820, doi: 10.1080/0305215X.2011.613465.

[7] Schrijver, A. (2003). Combinatorial Optimization: Polyhedra and Efficiency, Springer, Berlin, Germany. 
[8] Soleimani, H., Kannan, G. (2015). A hybrid particle swarm optimization and genetic algorithm for closed-loop supply chain network design in large-scale networks, Applied Mathematical Modelling, Vol. 39, No. 14, 39904012, doi: 10.1016/j.apm.2014.12.016.

[9] Coello Coello, C., Lamont, G.B., Van Veldhuizen, D.A. (2007). Evolutionary algorithms for solving multi-objective problems, Second edition, Springer, Berlin.

[10] Lai, Y.-J., Hwang, C.-L. (1992). A new approach to some possibilistic linear programming problems, Fuzzy Sets and Systems, Vol. 49, No. 2, 121-133, doi: 10.1016/0165-0114(92)90318-X.

[11] Pasandideh, S.H.R., Niaki, S.T.A., Asadi, K. (2015). Bi-objective optimization of a multi-product multi-period three-echelon supply chain problem under uncertain environments: NSGA-II and NRGA, Information Sciences, Vol. 292, 57-74, doi: 10.1016/i.ins.2014.08.068.

[12] Zeballos, L.J., Gomes, M.I., Barbosa-Povoa, A.P., Novais, A.Q. (2012). Addressing the uncertain quality and quantity of returns in closed-loop supply chains, Computers \& Chemical Engineering, Vol. 47, 237-247, doi: 10.1016/j. compchemeng.2012.06.034.

[13] Amin, S.H., Zhang, G. (2013). A multi-objective facility location model for closed-loop supply chain network under uncertain demand and return, Applied Mathematical Modelling, Vol. 37, No. 6, 4165-4176, doi: 10.1016/i. apm.2012.09.039.

[14] Hasani, A., Zegordi, S.H., Nikbakhsh, E. (2012). Robust closed-loop supply chain network design for perishable goods in agile manufacturing under uncertainty, International Journal of Production Research, Vol. 50, No. 16, 4649-4669, doi: 10.1080/00207543.2011.625051.

[15] Ramezani, M., Bashiri, M., Tavakkoli-Moghaddam, R. (2013). A new multi-objective stochastic model for a forward/reverse logistic network design with responsiveness and quality level, Applied Mathematical Modelling, Vol. 37, No. 1-2, 328-344, doi: 10.1016/j.apm.2012.02.032.

[16] Vahdani, B., Tavakkoli-Moghaddam, R., Jolai, F., Baboli, A. (2013). Reliable design of a closed loop supply chain network under uncertainty: An interval fuzzy possibilistic chance-constrained model, Engineering Optimization, Vol. 45, No. 6,745-765, doi: 10.1080/0305215X.2012.704029.

[17] Sherafati, M., Bashiri, M. (2016). Closed loop supply chain network design with fuzzy tactical decisions, Journal of Industrial Engineering International, Vol. 12, No. 3, 255-269, doi: 10.1007/s40092-016-0140-3.

[18] Wang, H.-F., Hsu, H.-W. (2010). A closed-loop logistic model with a spanning-tree based genetic algorithm, Computers \& Operations Research, Vol. 37, No. 2, 376-389, doi: 10.1016/i.cor.2009.06.001.

[19] Pishvaee, M.S., Kianfar, K., Karimi, B. (2010). Reverse logistics network design using simulated annealing, The International Journal of Advanced Manufacturing Technology, Vol. 47, No. 1-4, 269-281, doi: 10.1007/s00170009-2194-5.

[20] Soleimani, H., Seyyed-Esfahani, M., Shirazi, M.A. (2013). Designing and planning a multi-echelon multi-period multi-product closed-loop supply chain utilizing genetic algorithm, The International Journal of Advanced Manufacturing Technology, Vol. 68, No. 1-4, 917-931, doi: 10.1007/s00170-013-4953-6.

[21] Khalilpourazari, S., Mohammadi, M. (2016). Optimization of closed-loop supply chain network design: A water cycle algorithm approach, In: 12th International Conference on Industrial Engineering (ICIE 2016), Kharazmi University, Tehran, Iran, doi: 10.1109/INDUSENG.2016.7519347.

[22] Liang, T.-F., Cheng, H.-W. (2009). Application of fuzzy sets to manufacturing/distribution planning decisions with multi-product and multi-time period in supply chains, Expert Systems with Applications, Vol. 36, No. 2, Part 2, 3367-3377, doi: 10.1016/j.eswa.2008.01.002.

[23] Hwang, C.-L., Yoon, K. (1981). Multiple attribute decision making: Methods and applications: A state-of-the-art survey, Springer-Verlag, Berlin, Heidelberg, Germany, doi: 10.1007/978-3-642-48318-9.

[24] Deb, K., Pratap, A., Agarwal, S., Meyarivan, T. (2002). A fast and elitist multiobjective genetic algorithm: NSGA-II, IEEE Transactions on Evolutionary Computation, Vol. 6, No. 2, 182-197, doi: 10.1109/4235.996017.

[25] Al Jadaan, O., Rao, C.R., Rajamani, L. (2006). Parametric study to enhance genetic algorithm performance, using ranked based roulette wheel selection method, In: International Conference on Multidisciplinary Information Sciences and Technology (InSciT2006), Merida, Spain, Vol. 2, 274-278.

[26] Srinivas, N., Deb, K. (1994). Multi objective optimization using non-dominated sorting in genetic algorithms, Evolutionary Computation, Vol. 2, No. 3, 221-248, doi: 10.1162/evco.1994.2.3.221.

[27] Deb, K. (2001). Multi-objective optimization using evolutionary algorithms, Wiley, Hoboken, USA.

[28] Al Jadaan, O., Rajamani, L., Rao, C.R. (2009). Non-dominated ranked genetic algorithm for solving constrained multi-objective optimization problems, Journal of Theoretical and Applied Information Technology, Vol. 5, 714725. 